Rhode Island College

Digital Commons @ RIC

Master's Theses, Dissertations, Graduate

Master's Theses, Dissertations, Graduate

Research and Major Papers Overview

Research and Major Papers

2010

\title{
A Program Evaluation of a Nurse-led Interdisciplinary Heart Failure Clinic
}

Victoria Lee Reis Savard

Rhode Island College

Follow this and additional works at: https://digitalcommons.ric.edu/etd

Part of the Nursing Commons

\section{Recommended Citation}

Reis Savard, Victoria Lee, "A Program Evaluation of a Nurse-led Interdisciplinary Heart Failure Clinic" (2010). Master's Theses, Dissertations, Graduate Research and Major Papers Overview. 85.

https://digitalcommons.ric.edu/etd/85

This Major Paper is brought to you for free and open access by the Master's Theses, Dissertations, Graduate Research and Major Papers at Digital Commons @ RIC. It has been accepted for inclusion in Master's Theses, Dissertations, Graduate Research and Major Papers Overview by an authorized administrator of Digital Commons @ RIC. For more information, please contact digitalcommons@ric.edu. 


\title{
A PROGRAM EVALUATION OF A NURSE-LED \\ INTERDISCIPLINARY HEART FAILURE CLINIC
}

A Major Paper Presented

\author{
By
}

Victoria Lee Reis Savard

Approved:

Committee Chairperson

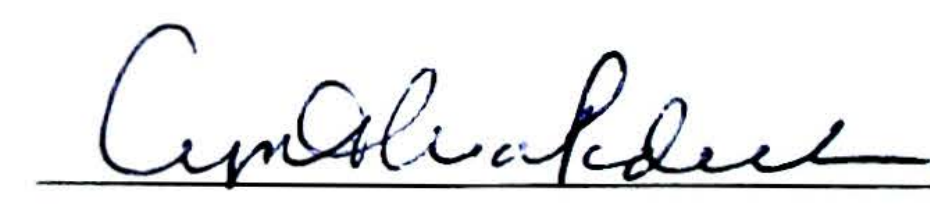

$5 / 11 / 10$

Committee Members

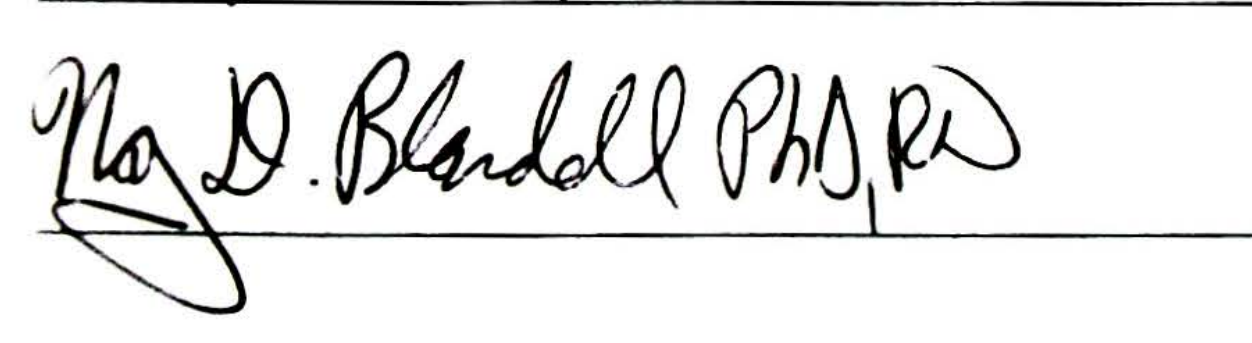

$\frac{5 / 10 / 10}{\text { (Date) }}$

Director of Master's Program

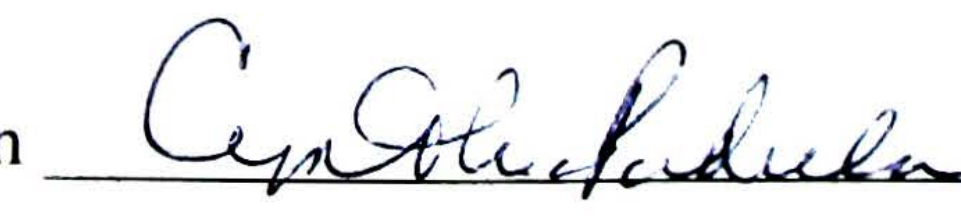

Dean, School of Nursing

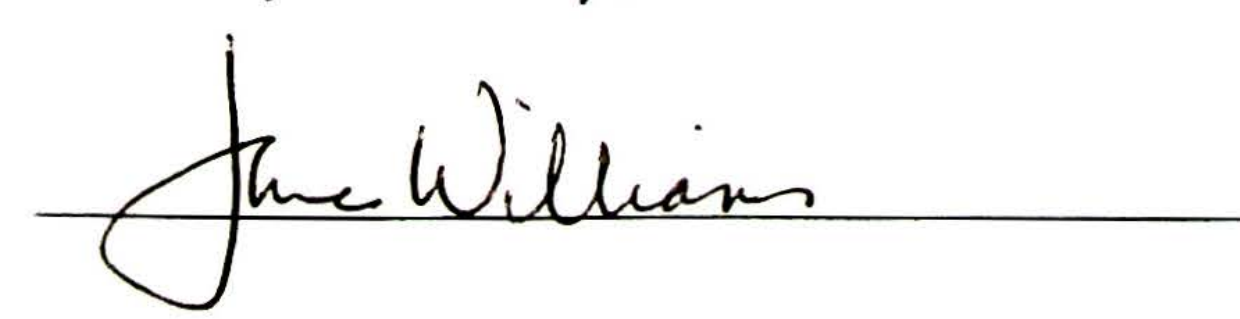

$5 / 11 / 10$

(Date)

$5 / 20 / 10$

(Date) 
Victoria Lee Reis Savard

\author{
A Major Paper Submitted in Partial Fulfillment \\ of the Requirements for the Degree of \\ Master of Science in Nursing \\ in
}

The School of Nursing

Rhode Island College

2010 
Abstract

Heart failure (HF) is a major health disparity that accounts for a vast number of hospitalizations as well as re-hospitalizations. In 2006. the estimated direct cost of HF in the United States (US) was 29.6 billion dollars (American Heart Association [AHA], 2005). HF is not only costly but it also accounts for approximately 287,000 deaths in the US each year (AHA). Significant improvements in patient outcomes are evident when patients are referred to HF clinics. From the literature it appears that referred clients have less frequent re-hospitalizations in addition to improved quality of life. Nurse-led outpatient HF clinics offer behavioral specific knowledge necessary to engage in health promotion. Due to the complexity of the disease, it is appropriate to refer clients diagnosed with HF to nurse-led outpatient HF clinics. The purpose of this project was to validate and possibly expand knowledge about specific aspects of the nursing role in particular that contribute to improved outcomes in nurse-led HF clinics. A nurse interview and nurse-client observations were performed with specific emphasis on the role of the nurse. Scores were assessed on five clients using the Minnesota Living with Heart Failure questionnaire. Each demonstrated improvement on questionnaire responses during the six month period, with scores varying in improvement from 10-12 points at the six month mark. Statistics related to re-hospitalization rates and ER visits were provided for the program evaluation by the clinic. The HF clinic demonstrated a 30 day rehospitalization rate less than the national average of $24 \%$, with a percentage of $18.6 \%$. Components of a successful program were observed during an evaluation of the program. Recommendations and implications for advanced practice are discussed. 
Table of Contents.

Problem Statement 1

Literature Review

3

Theoretical Framework ......................................................................

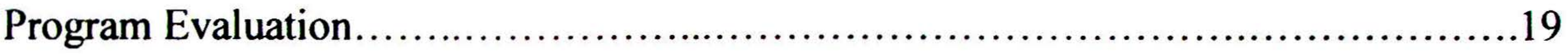

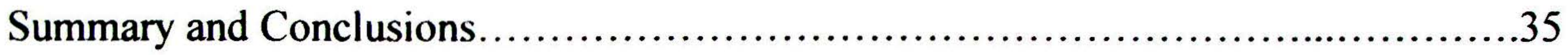

Recommendations and Implications for Advanced Practice ..........................37

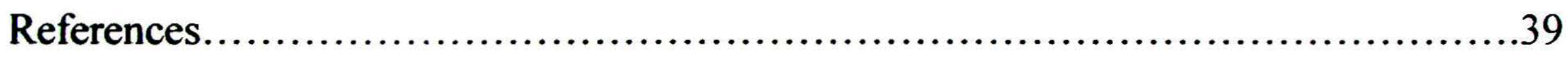

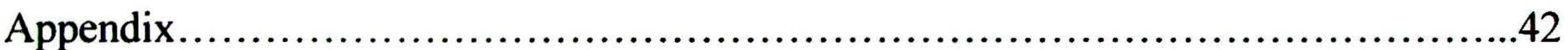


A Program Evaluation of a Nurse-led Interdisciplinary Heart Failure Clinic

\section{Problem Statement}

Heart failure (HF) is an ongoing health problem in the United States (US) and the world. The American Heart Association (AHA) Task Force reported that approximately 5 million Americans have HF and more than 550,000 patients are newly diagnosed with HF each year (AHA, 2005). Patients with HF face a substantial risk for recurrent exacerbations. The Centers for Medicare and Medicaid Services (CMS, 2009) reported that the US national rate for HF patients readmitted within 30 days is currently $24.5 \%$. $\mathrm{HF}$ is the most common cause of hospitalization due to cardiovascular disease in patients more than 65 years of age and is associated with frequent readmission within 30 days (Cline, Brom, \& Wittenberg, 1996). People with HF experience symptoms such as dyspnea, edema, weight gain, and fatigue which can limit activity tolerance and quality of life (AHA, 2005). Since HF is a chronic condition, most lifestyle change is made on an outpatient basis, necessitating follow-up in regard to medication effectiveness and symptom monitoring. Behavioral factors, such as poor compliance with treatment, frequently contribute to exacerbations of $\mathrm{HF}$, a fact suggesting that many admissions could be prevented (Rich et al., 1995). HF clinics are designed to enhance follow-up surveillance, improve compliance, and reinforce patient education. Yet, patients are not always referred to clinics following discharge from acute care hospitals. HF clinics are considerably diverse but on the other hand, exceedingly similar, as evidenced from a review of the literature. Although clinics are managed by an array of specialized clinicians, each clinic has the same objective: to reduce hospitalization rates and associated costs. Nurse-led HF clinics are effective and have demonstrated a decrease in 
re-hospitalization rates amongst their referred clients. The purpose of this project was to evaluate the role of the nurse in a nurse-led HF clinic and to assess what components allow for successful outcomes. 


\section{Literature Review}

The literature review included studies which evaluated the effects of HF programs on hospitalization rates and/or quality of life. A systematic search of PubMed and CINAHL (1992-2009) was performed with specific interest on the key terms: outpatient HF programs; multidisciplinary care; interdisciplinary care; nurse-led clinics; rehospitalizations; readmissions; and quality of life. The focus on the literature included studies which evaluated multidisciplinary and nurse-led outpatient HF clinics. The reference list of the reviewed articles was also examined and reviewed.

\section{Background}

The AHA (2005) defined HF as a complex clinical syndrome that can result from any structural or functional cardiac disorder that impairs the ability of the ventricle to fill with or eject blood. The cardiac dysfunction results in insufficient cardiac output. HF can be caused by an array of disorders including coronary artery disease, hypertension, cardiomyopathy, congenital heart defects, valvular disorders, and hyperthyroidism. Regardless of the etiology, clients presenting with HF may experience similar symptoms such as dyspnea, fatigue and even peripheral edema. The AHA reported that HF is a chronic, progressive disease that is characterized by frequent hospital readmissions and ultimately high mortality rates. Patients with $\mathrm{HF}$ are at increased risk for rehospitalization as discussed in the literature. Perhaps, the clients' symptoms are to blame. These symptoms have an impact on the client's functional status and quality of life (AHA, 2005). The treatment of HF is aimed at improving cardiac output while decreasing cardiac workload and minimizing symptoms (Copstead \& Banasik, 2000). Along with pharmacological treatments, clients with HF must receive education on the disease 
process including signs and symptoms and prevention of HF (AHA. 2005). Symptom management through education can be provided by referrals to outpatient HF programs in the community, In order to gain a better understanding of HF programs, in hospital, multidisciplinary and nurse-led programs were reviewed in the literature.

\section{Inparient HF Clinics with Outpatient Component}

Some of the research focused on nurse-led education initiated prior to discharge from a hospital facility. In a prospective randomized trial conducted by Rich et al. (1995). ficus was placed on inpatient education and outpatient follow-up. The study purpose was to examine the effect of a nurse-directed. multidisciplinary intervention on rates of readmission within 90 days of hospital discharge. quality of life. and costs of care for high-risk patients 70 years of age or older who were hospitalized with HF. The intervention group was provided with comprehensive education, a prescribed diet, socialservice consultation, a review of medications. and intensive follow-up after discharge. The outpatient component played an important role in the research, and post discharge services included home care services, supplemented by home visits and telephone contact by an experienced cardiovascular research nurse. The control group received all standard treatments and services ordered by their primary care providers (PCPs).

In 90 days. the number of readmissions for HF was reduced by $56.2 \%$ in the treatment group (54 readmissions $T$ vs. 24 readmissions $C ; p=.04$ ). In the intervention group. 91 of the $142(64 \%)$ patients were without readmission in the 90 days. as compared with 75 of the $140(53 \%)$ patients in the control group. who received conventional care. In a subgroup of 126 patients. quality-of-life as measured by the Chronic Heart Failure questionnaire scores at 90 days improved more from baseline for patients in the treatment 
group $(22.1 \pm 20.8 \mathrm{~T}$ vs. $11.3 \pm 16.4 \mathrm{C} ; \mathrm{p}=.001)$. This study concluded that a nursedirected, multidisciplinary intervention can improve quality of life and reduce hospital use and medical costs for elderly patients with HF.

In a study by McDonald et al. (2002), education began on an inpatient basis and continued with follow-up at an outpatient clinic. The purpose of this study was to address the unanswered question of whether multidisciplinary care of HF can reduce readmissions when optimal medical care is applied in both intervention and control groups. In this randomized, controlled study, 98 patients admitted to hospital with HF were assigned to routine care which was comprised of outpatient follow-up with their PCP $(n=47)$ or multidisciplinary care $(n=51)$. All patients received the same components of inpatient care. Education provided by inpatient nursing staff included daily weight monitoring, disease and medication understanding, and salt restriction. Information was provided to the patient and caregiver. The intervention group was referred to the PCPs for outpatient follow-up. Those randomly selected into the control group received inpatient and outpatient education with close telephone contact and clinic follow-up. Multidisciplinary care was initiated with a close clinic follow-up by nurse telephone contact at three days post discharge and weekly thereafter (for education and diuretic treatment adjustment as per protocol). At weeks two and six, patients and their next of kin attended the HF clinic to check clinical status and further revise key education issues by the nurse. The nurse was described as a HF specialist nurse with no specific description provided regarding educational background. Supervising cardiologists were readily available to nurses for consultation. The outpatient component consisted of HF specific education provided to patients and families utilizing videotapes, group education 
sessions and printed materials on HF. Patients were encouraged to self-monitor symptoms, assess daily weights, and consider dietary restrictions. The primary outcome variable was $\mathrm{HF}$ re-admission. HF re-admission was far less frequent in the intervention group ( $25.5 \%$ readmission $C$ vs. $3.9 \%$ readmission I). Those receiving multidisciplinary care were at far less probability of hospital readmission.

\section{Multidisciplinary HF Clinics}

Cline. Israelsson. Willenheimer. Broms and Erhardt (1998) conducted a prospective randomized trial to study the effects of a management program on hospitalization and health care costs one year after admission for HF. In this study, the term management program was never specified. The study randomized 190 patients ages $65-84$ years hospitalized with $\mathrm{HF}$ to an intervention group assessed by specially trained registered nurses or to a control group. These management programs were led by nurses said to have HF experience from working in coronary care units and/or in clinical HF trials and from attending HF lectures. The control group received usual care which included follow up by either private practice cardiologists or by their PCPs. The intervention group received education on $\mathrm{HF}$ and self management; with follow up at a nurse outpatient clinic for one year after discharge. The education program included the pathophysiology of $\mathrm{HF}$ and pharmacological and non pharmacological treatment. Clients also received guidelines for self management of diuretics based on worsening signs and symptoms of HF. Nurses were available via telephone during office hours as well as available to see clients on a short notice. The control group was managed according to routine clinical practice and the $\mathrm{PCP} /$ cardiologist was free to evaluate and treat the client as they deemed appropriate. 
Time to first readmission over the same period was $33 \%$ longer in the intervention group (106 days $\mathrm{C}$ vs. 141 days I; $\mathrm{p}<.05$ ). Researchers also reported a trend towards reduced number of admissions and days in hospital in the intervention group. Hospitalization one year prior to the study was compared with hospitalization during the study period. During one year follow-up, there was an increase (59\%) in the number of days hospitalized in the control group (5.1 [10.6] mean days prior to study C vs. 8.1 [14.3] days during study $C ; p<.05$ ). There was no increase in the intervention group (4.2 [7.9] mean days prior to study I vs. (4.3 [7.8] days during study I). The Cline et al. study adds some evidence in support of nurse led multidisciplinary intervention following admission to hospital with HF. The study concluded that a management program for patients with HF discharged after hospitalization reduces readmission rates as well as health care costs. The lower readmission rate in the intervention group contributed to a mean annual reduction in the overall costs of US $\$ 1300$ per patient $(p=.07)$. This was the result of the decreased number of readmissions.

In a prospective randomized trial conducted by Kasper et al. (2002), 200 patients hospitalized with HF with increased risk of hospital readmission were unsystematically referred to a multidisciplinary program or to follow-up with their PCPs. Prior to hospital discharge, a baseline evaluation including a history and physical examination performed by cardiologists specialized in the management of HF was conducted along with medication and dietary recommendations. These were documented in the medical records before randomization to the interventional or control groups. The treatment group was referred to a HF clinic while the control group received treatment on the discretion of their PCP. 
The multidisciplinary team consisted of a telephone nurse coordinator, the HF nurse, the HF cardiologist and the patient's PCP. For those placed in the intervention group, telephone calls were placed within 72 hours of hospital discharge, then weekly for one month, twice in the second month and monthly thereafter, unless a problem occurred that required more frequent contact. The 'telephone' nurse pursued problems but did not adjust medications over the telephone. The 'telephone nurse' made 973 calls to patients in the intervention group, averaging 9.5 calls per patient. As discussed in the study, nurses supervised by cardiologists, along with PCPs, provided most of the care, adjusted medications, and provided dietary and physical activity guidance. The HF nurses implemented the therapeutic plan designed by the HF cardiologists. Patients had at least monthly follow-up with these nurses at HF clinics and some at clients' homes. The HF nurses made 862 patient visits, or 8.5 visits per patient. The mean duration of a telephone call was $16 \pm 9$ minutes, whereas the average length of a visit by a HF nurse was $57 \pm 21$ minutes. Educational background and credentials of nurses were not discussed in the study. The HF nurses were able to adjust medications under the directions of the HF cardiologists, following a pre-specified algorithm. The 55 page algorithm, developed by the HF cardiologists using HF guidelines and clinical experience, incorporated a detailed description of the medication initiation and titration, monitoring of medical, dietary and activity therapies including a 2-g sodium-restricted diet as well as a recommendation to exercise by walking for 20 minute at least four days per week. The treatment plan was individualized for each patient. The HF cardiologists designed and documented a treatment plan. The primary physicians managed all problems not related to HF, received regular updates from the HF nurses, and were notified of abnormal laboratory values. The 
intervention group was supplied with a pill sorter, a list of correct medications, a list of dietary and physical activity recommendations, a contact number available 24 hours per day and patient education material.

The study assessed the primary outcome variable of death from any cause and the total number of HF hospital admissions. The results determined 59 hospital admissions for HF among 35 patients in the nonintervention group, and 43 hospital admissions among 26 patients in the intervention group $(\mathrm{p}=.09$ by the $\log$-transformation $t$ test and $\mathrm{p}=.03$ by the Poisson model comparison). The intervention patients were also more likely to be at their goal weight, as compared with the nonintervention patients (47of 94 patients vs. 17 of 85 patients, $p=.001)$. The study also determined that patients in the intervention group were more likely to report stable or improved symptoms, as compared with those in the nonintervention group ( 81 of 94 patients vs. 55 of 85 patients, $p=.003$ ) and were less likely to have ankle edema (18 of 89 patients vs. 35 of 85 patients, $p=.003$ ). Kasper et al. concluded that a multidisciplinary approach to the management of high-risk outpatients with $\mathrm{HF}$, utilizing an expert knowledge algorithm, frequent monitoring, intensive and continuing patient education, and close interaction with the patients' primary physician improved quality of life. The authors also noted a trend toward improvement in the primary end point of death and total number of HF hospital admissions over a six-month intervention period. Due to the comprehensive nature of the intervention, it was difficult to determine what aspects of the multidisciplinary program allow for improved outcomes.

Similar findings were reported by Ducharme, Doyon, White, Rouleau, and Brophy (2005), who conducted a randomized study with 230 eligible patients who had experienced an acute episode of HF. They compared standard care $(n=115)$, which was 
according to what was prescribed by the attending physician, with treatment at a multidisciplinary specialized HF outpatient clinic $(n=115)$. The intervention consisted of a structured outpatient clinic environment with complete access to cardiologists, nurses, and allied health professionals. The standard of care for the intervention group included a one on one educational session with a clinician nurse which was initiated at the first clinic visit and an individualized treatment plan by a clinic cardiologist. Similar to the previous study, detailed information about nurse education and training was lacking. These educational sessions consisted of a detailed explanation of the disease process, signs and symptoms of $\mathrm{HF}$, fluid and sodium restriction, the importance of daily weight monitoring, medication compliance, and a recommendation regarding exercise and diet. An educational booklet produced in-house entitled Living with Heart Failure was also provided to the clients. A reinforcement of this individualized educational program was provided at each subsequent visit.

The study focused on primary outcomes of all-cause hospital admission rates, total number of days in hospital at six months, as well as secondary outcomes of total number of emergency department visits, quality of life, and total mortality. At the six month mark, the intervention group had fewer patients that required re-hospitalization than the control group (45 [39\%] days I vs. 66 [57\%] days C). The subjects in the intervention group stayed in hospital for 514 days as compared to 815 days required by patients in the control group (adjusted HR 0.56, 95\% CI 0.35-0.89). Numbers of emergency department visits were similar in both the intervention and control groups. Quality of life, which was self-assessed using the Minnesota Living with Heart Failure questionnaire, was unchanged in the control group but improved in the intervention group 
$(p<.001)$. No significant difference in mortality was observed, with 19 deaths in the control group and 12 in the intervention group (HR $0.61,95 \% \mathrm{CI} 0.24-1.54)$. There was an improved clinical trend in mortality rates amongst the intervention group. The findings indicated that compared with usual care, care at a multidisciplinary specialized HF outpatient clinic reduced the number of hospital readmissions and hospital days and improved quality of life.

\section{Nurse-led HF Clinic}

Stromberg et al. (2003) conducted a prospective randomized trial which evaluated the effect of follow-up for 12 months after discharge at a nurse-led HF clinic on mortality, morbidity and self-care behavior for patients hospitalized due to HF. The study included a total of 106 patients randomly assigned to either follow-up at a nurse-led HF clinic or to usual care. Clients referred to the usual care practice were followed up by their PCPs and managed in accordance with clinical guidelines and the PCPs' clinical judgment. The nurse-led HF clinic was staffed by specially trained and experienced cardiac nurses. Neither the educational level nor the experience level of the nurses were specified and can be considered a limitation of the study. The first visit was scheduled two to three weeks after discharge. The nurse evaluated the patient status, results of treatment, and provided education about $\mathrm{HF}$ and social support to the patient and family during the one hour visits. The education was individualized and included both written and verbal information. The clients and their families were educated on HF with specific content including: the definition and signs/symptoms of HF; etiology; treatment rationale; drug counseling; non-pharmacological treatment; and dietary influences on HF. The patients could contact the clinic during daily telephone hours. Trained nurses called patients in 
order to provide psychosocial support, evaluate drug changes, in response to calls placed by patients, or other actions taken due to deterioration and side effects. The frequency of the follow-up calls was not included in the study.

There were fewer patients with negative events (death or admission) after 12 months in the intervention group compared to the control group ( 29 admissions/death I vs. 40 admissions/death C: $p=.03$ ) and fewer deaths after 12 months ( 7 deaths I vs. 20 deaths C; $p=.005$ ). After 12 months, the intervention was associated with a $55 \%$ decrease in admissions per patient per month ( $0.18 \mathrm{admissions} /$ patient/month I vs. 0.40 admissions/patient/month $C ; p=.06)$ and fewer days in hospital per patient per month (1.4 hospital/patient/month I vs. 3.9 hospital/patient/month $\mathrm{C} ; p=.02$ ). The intervention group had significantly higher self-care scores at $3(\mathrm{p}=.02)$ and 12 months $(p=.01)$ compared to the control group. The study concluded that follow up after hospitalization at a nurse-led HF clinic can improve survival and self-care behavior in patients with HF as well as reduce the number of events, readmissions and days in hospital. Nurse led clinics clearly demonstrated a positive outcome.

After reviewing the scientific literature, the conclusion is that nurse-led multidisciplinary outpatient clinics appear to reduce the re-hospitalization rates as well as improve quality of life. The evidence also supports a multi faceted approach that incorporates educational and supportive needs provided in nurse-led clinical settings.

The question still remains: What components of the nursing role attribute to a successful nurse-led HF clinic? 


\section{Theoretical Framework}

The Health Promotion Model (HPM) provided the organizing framework for this program evaluation. The HPM (Figure 1), developed by Nola J. Pender in1982 and revised in 2006, was designed to integrate nursing and behavioral science perspectives on factors that influence health behaviors. The model (2006) defines health as a positive dynamic state not merely the absence of disease. The HPM includes components of health promoting behaviors which are categorized into individual characteristics and experiences, behavior specific cognitions and affects and the overall behavioral outcome. Pender's framework proposed that the acquisition and maintenance of health-promoting behavior depends upon these three components. Pender (2006) defined health-promoting behaviors as patterns of self actions and perceptions which serve to maintain wellness, self- actualization, and fulfillment of the individual. This model posits that each person has unique personal characteristics and experiences which play a role in actions and health promoting behaviors.

The first phase of the HPM contains characteristics and experiences which are unique to the individual. Individual characteristics indirectly influence health-promoting behaviors. This category is subdivided to focus on the individual's prior related behaviors and personal factors. A person's prior experiences with a given activity may influence the person's participation in the activity. Prior related behaviors with negative experiences hinder the likelihood that an individual will participate in the health promoting behavior. Personal factors such as biological, psychologic, or sociocultural factors also play a role in this category. Personal biological factors include variables such as; age, gender, body mass index, strength or balance. Personal psychological factors may include self 
INDIVIDUAL

CHARACTERISTICS

AND EXPERIENCES
BEHAVIOR-SPECIFIC

COGNITIONS

AND AFFECT
BEHAVIORAL

OUTCOME

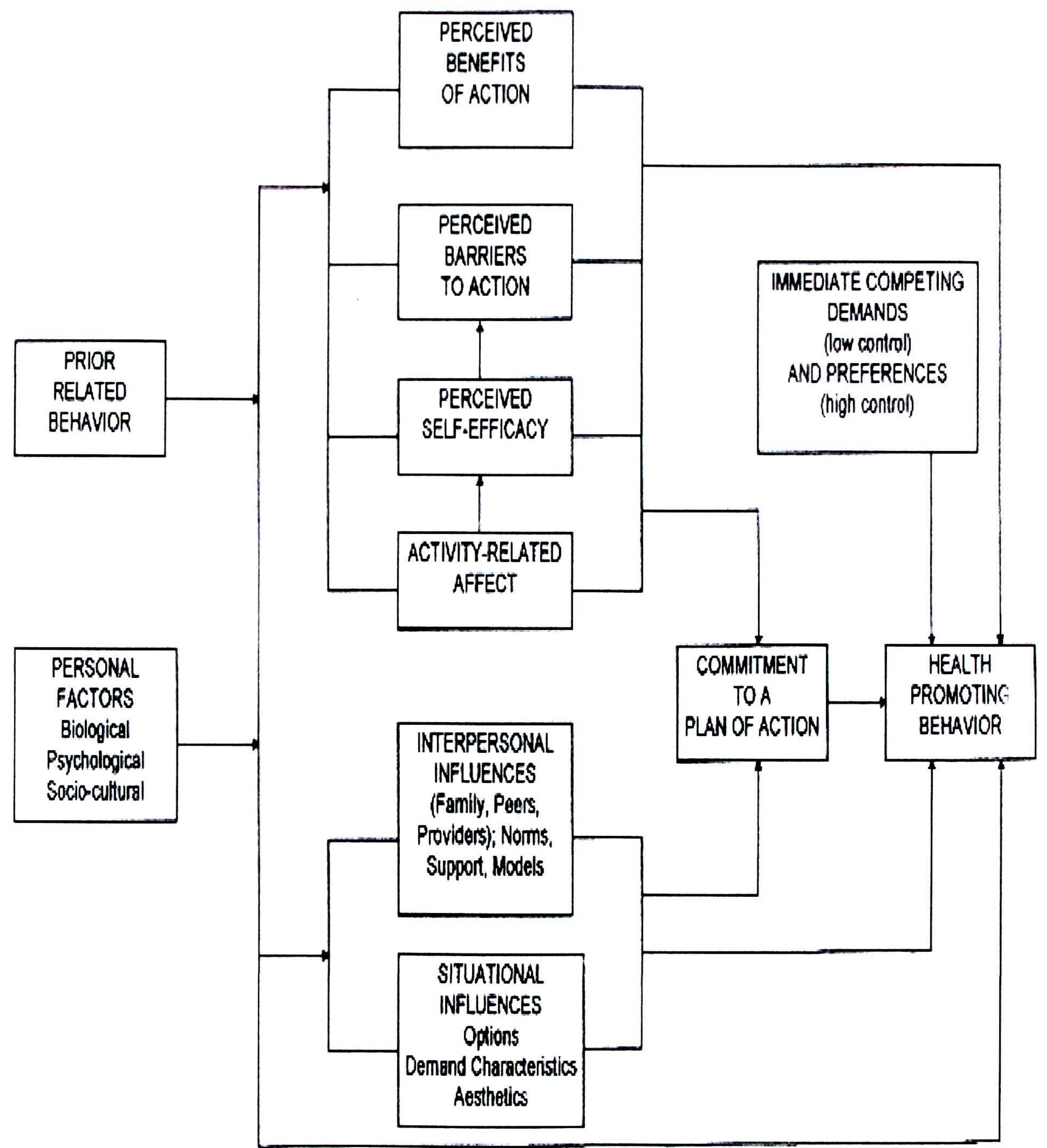

Revised Health Promotion Model

Figure 1. Health Promotion Model 
motivation, perceived health status and the individual's personal definition of health. Age, gender, income, ethnic, racial, socioeconomic status, and educational background comprises the personal socio-cultural factors associated with the model (Pender 2006).

The second phase in the HPM focuses on the behavioral specific cognitions and affect. These include six areas: perceived benefits of action: perceived barriers to action; perceived self efficacy; activity related affect; interpersonal influences; and situational influences (Pender, 2006). These are considered to be the primary mechanisms directly affecting the chance of adopting health-promoting behaviors. Perceived barriers and benefits also have an impact on health-promoting behavior. Benefits are considered the person's incentive which may be internal or external. An example of an internal incentive can be the belief of improved health status. An external incentive may be compensation provided by a caregiver or program for completing a health-promoting behavior. Barriers may be what the client perceives as a negative impact to the behavior. If a person believes the side effects to a medication may outweigh the benefits of taking the medication, the person may be less likely to comply with the medication regimen. Perceived self-efficacy is the person's beliefs about their capabilities to perform a specific behavior. Self-efficacy is sometimes seen as the motivating factor. The person's perceived health and definition of health have an impact on the person's likelihood to perform a behavior or make a change. If the person believes their health status is not influenced by behavior modification, they are least likely to perform or make a suggested change. Activity related affects are the positive or negative feelings that occur before, during, and following the health promoting behavior. These can influence perceived selfefficacy which can generate a positive effect. 
Interpersonal influences relate to norms, model, social support and the expectations of significant others. Family members, peers, and other influential caregivers can provide encouragement or discouragement toward a change of behavior.

Situational factors influence behavior through the surrounding environment. A person can be affected by his/her surroundings as well as their surroundings may affect their ability to change. A client with HF may not be able to control the diet provided to them, which may impact their sodium restricted diet, in turn impacting their HF. Other situational factors may include perceptions of options available, demand characteristics and aesthetic features of the environment in which given health promoting is proposed to take place (Pender, 2006)

The last phase of the HPM is the behavioral outcome, which is influenced by the relationship between individual characteristics and experiences and behavioral specific cognitions and affect. It combines the commitment to the action plan, the competing demands and preferences, with the final component, that of the health promoting behavior. The commitment to plan of action is based on the concept that the intention and identification of a planned strategy leads to implementation of health behavior. Competing demands reflect the alternative behavior over which individuals have low control because there are environmental contingencies. These may include work or even family care responsibilities. Competing preferences are of higher control and may include the persons' ability to choose fruit over a piece of cake. The health promoting behavior encompasses the action directed toward achieving the positive health promoting behavior. Health promoting behavior is the desired behavioral outcome and is the end point in the HPM. Health promoting behaviors should result in improved health, 
enhanced functional ability and better quality of life at all stages of development (Pender, 2006).

Each client is unique, not only in terms of level of HF, but also in terms of his or her personal and cultural approach to the disease. The HPM takes into consideration that each individual is unique in characteristics and experiences and that the individual is ultimately responsible for engaging in a health promoting behavior. Behavioral specific knowledge and affect have important motivational significance and can be modified by nursing actions. Nurse-led HF clinics are a type of health promotion programs that can result in improved health, enhanced functional ability, and better quality of life. In this model, an individual performs a health behavior based on perceived benefits, perceived barriers, and perceived self-efficacy (Pender, 1974). Personal factors, including biological, psychological, and socio-cultural variables, are believed to play an important role as they are predictive of a given behavior. Perceived benefits and barriers relate to the psychologic components whereby individuals evaluate the results related to a health promotion behavior such as the specific activities the client undertakes at a HF clinic. Perceived barriers can constrain commitment to action, a mediator of behavior, as well as actual behavior (Pender, Murdaugh, \& Parsons, 2002). Consequences of the risk and condition must be specified as well as action to take. In the case of the person with HF, taking medications or following a low-salt diet may reduce the progression of symptoms and be seen as beneficial. These benefits help to weigh against perceived barriers that the client may face. Perceived barriers are the potential negative consequences of a certain health behavior. In clients with HF, a barrier to following a low sodium diet recommendation might be the outcome of taste dislike caused by no added salt. In this 
ease, the person with HF would weigh risks and benefits betore making a decision about whether to follow a recommended course of action. Lack of compliance coupled with inadequate plans of eare can lead to increased hospitalizations. In order to motivate the Slient to comply with $\mathrm{HF}$ treatment. education must he reinforced. Education must be based on personalized risk and hehavior. Clients should also be explained potential barriers and ways to reduce them through reassurance, incentives, and assistance. By providing assurance of positive effects to be expected. clients are more likely to comply with the treatment.

Pender's HPM will the used in this project to examine health promoting activities used in the clinic. Clients will he evaluated with specific attention to individual characteristics. behavioral cognitions and behavioral outcomes.

Next. the program evaluation process will be presented and discussed. 


\section{Introduction}

\section{Program Evaluation}

HF clinics provide intensive outpatient management to improve health status, prevent clinical deterioration, and avert acute crisis. This project took place at a local cardiac practice in northern Rhode Island. Clients seen at the clinic have a primary diagnosis of HF including both newly diagnosed as well as chronic. Currently, the chronic HF patients at the clinic are seen on a monthly basis; the more acute patients are seen weekly or bimonthly, depending on their symptoms. Patients newly diagnosed with HF have an initial visit with the physician then meet with a nurse for a one hour education session which includes the family or a significant other whenever possible. In the event that a patient is assessed as having a need for further education, then additional time is provided to the client.

With their vested interest in the evaluation of the clinic, responsible administrators agreed to participate in the program evaluation. The plan for evaluating the HF clinic was approved by agency administrators as well as the educational institution's IRB.

\section{Purpose}

The purpose of this project was to evaluate the effects of a nurse-led interdisciplinary heart failure clinic and to determine the nurse's role as derived from observation of the interactions that occur with their clients. The overall evaluation goals of the project were both process and outcome based. In order to guide the program evaluation, the Centers for Disease Control (CDC) framework was utilized.

\section{The CDC Framework for Program Evaluation}

The CDC framework (Figure 2) is comprised of multiple components which include 
steps in evaluation practice and standards for effective evaluation (CDC, 1999). The steps and standards are simultaneously in the evaluation process.

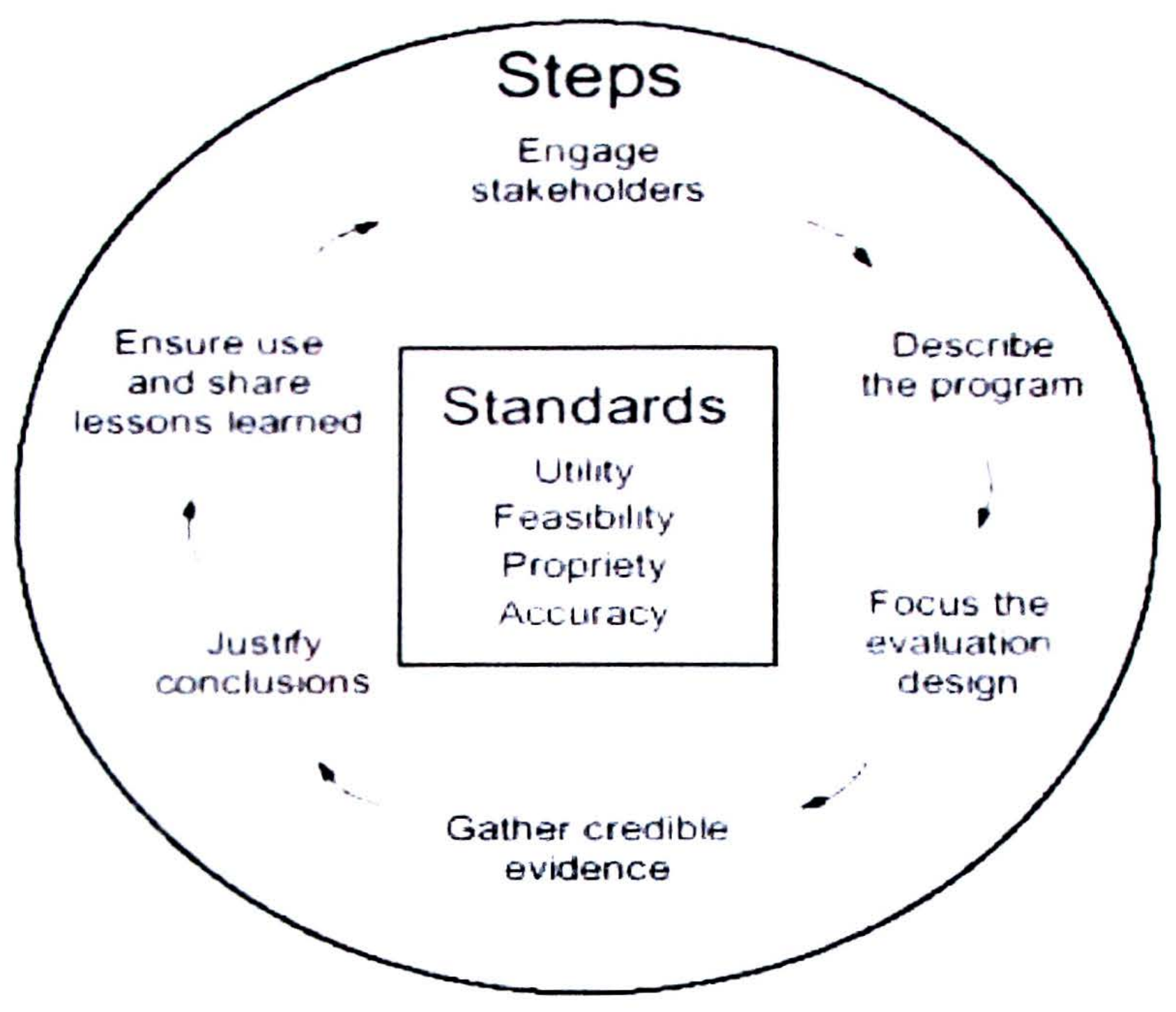

Figure 2. The CDC Framework for Program Evaluation

The six steps of the framework (Table 1) supply the structure to program evaluation.

These include engaging the stakeholders, describing the program, focusing on the evaluation design, gathering credible evidence, justifying conclusions and ensuring that the lessons are disseminated.

Table 1 .

Steps in the CDC Evaluation Practice

\section{Engage Stakeholders}

Describe the Program

Focus the Evaluation Design

Gather Credible Evidence

Justify Conclusions

Ensure Use and Share Lessons Learned
Those persons involved in or affected by the program, and primary users of the evaluation. Need, expected effects, activities, resources, stage, context, logic model.

Purpose, users, uses, questions, methods, agreements.

Indicators, sources, quality, quantity, logistics.

Standards, analysis/synthesis, interpretation, judgment, recommendations.

Design, preparation, feedback, follow-up, dissemination. 
The standards for effective evaluation (Table 2) are assessed by utility, feasibility, propriety and accuracy.

Table 2.

Standards for Effective Evaluation

Utility

Serve the information needs of intended users.

Feasibility

Propriety

Be realistic, prudent, diplomatic, and frugal. Behave legally, ethically, and with regard for the welfare of those involved and those affected.

Accuracy

Reveal and convey technically accurate information.

The initial phase of the evaluation cycle begins by engaging stakeholders. The stakeholders have an invested interest in the program evaluation. They may or may not have financial ties to the program. The stakeholders also assist with the execution of other steps. They assist with engaging others affected by the evaluation process. These may include those involved in program operations such as sponsors, administrators and/or staff members. It may also involve those served or affected by the program such as clients, support systems, staff members and/or primary users of the evaluation, which may include those persons involved the decision making position. The next step in the evaluation framework is to provide a description of the program. The description phase assesses the need of the program, its functional structure, and the goal and expectation of the program. Once the program has been described, the focus on the evaluation design may be performed. This entails planning the evaluation and the steps needed to initiate the process.

A thorough plan anticipates intended uses and creates an evaluation strategy with the greatest chance of being useful, feasible, ethical, and accurate (CDC, 1999). In each program evaluation, gathering credible evidence that conveys an overall representation of 
the program's statistics should be obtained. This allows program credibility. Once the data has been collected, evaluation conclusions are justified and are linked to the evidence gathered and judged against agreed-upon values or standards set by the stakeholders (CDC). The final step in the CDC framework encompasses dissemination of the evaluation procedures and findings to the stakeholders. This process should incorporate customized communications strategies that meet the stakeholder's specific needs.

Next, each step of the evaluation process will be discussed in relation to this program evaluation.

Engaging the stakeholders. The stakeholders involved with the HF clinic included the program director, a physician, the nurse leading the clinic, and the clients attending the clinic. The program evaluation took place at a cardiology practice in northern Rhode Island. The HF clinic is one program the practice supports. Clients are seen at the practice for multiple cardiac diagnoses including hypertension, atrial fibrillation, coronary artery disease and post pacemaker insertion follow up. A description of the different roles incorporated into the clinics' structure and function was reviewed.

The program director is the financial carrier of the program and also the chief physician of the practice. He oversees the clinic and has a vested interest in the program evaluation. There is also a physician employed by the program director who is available for triage or consultation. He provides care to cardiac clients at the office as well as consulting on admitted clients from the practice at local hospitals.

The nurse at the clinic provides assessment and educational support to the clients visiting the HF clinic. The title for the nurse at the clinic is the 'HF Clinic Coordinator/ 
Patient Educator'. She has been employed in this role for over 10 years. The HF Clinic Coordinator provides educational consultation to clients with atrial fibrillation receiving coumadin, clients receiving lipid-lowering agents, and the clients with a diagnosis of HF. Although her role at the clinic encompasses education to various cardiac patients, her main focus is with the HF clients. The HF Clinic Coordinator/Patient Educator has a Master's Degree in Nursing and has attended multiple conferences on HF. Prior to working at the clinic, the nurse was a telemetry/cardiac nurse at the local hospital. Her professional responsibilities are mainly to assess these clients at risk for re-hospitalization and evaluate their educational needs related to HF. The nurse's daily routine varies as one day she sees clients back to back and other days she follows-up on critical labs and diagnostic testing for these clients. Typically, clinic visits involve a one hour session with the nurse. Clients can also call the clinic during office hours with any questions or concerns. Follow-up phone calls are made to these clients by the nurse. A descriptive analysis of the program assisted the evaluator to focus on the purpose of the clinic and its' outcomes in relation to HF re-hospitalization rates and quality of life.

Describing the program. Since HF is a major health disparity, HF clinics are needed to assess symptoms and prevent re-hospitalization. The clinic is situated in a cardiology office and carries the same office hours of Monday through Friday from 9am-5pm. For off hours, the physician on call is available to these clients via telephone contact.

After a hospital admission for HF, clients are provided with follow up appointments prior to discharge from the local hospital. Those who have previously attended the clinic are provided with appointments based on HF status and symptoms at the discretion of the nurse. Clients at risk for re-hospitalization are seen more frequently. The goal of the 
clinic is to establish and maintain an excellent clinical service for patients with acute/chronic HF and to provide these clients with the medical and educational need to improve quality of life.

Evaluation of the client is initiated while the client is still in the waiting room. Any laboratory and diagnostic testing is reviewed by the nurse prior to the client's arrival to the clinic. Typically, clients obtain blood work monthly. Special circumstances may arise for clients with abnormal lab values, requiring more frequent blood work. Clients are assessed with the Minnesota Living with Heart Failure (MLHF) questionnaire (Rector \& Cohn, 1992) at each clinic visit and evaluated by the $\mathrm{RN}$ for signs and symptoms of $\mathrm{HF}$, including weight gain, shortness of breath, edema, and activity intolerance. The questionnaire is provided at check-in and completed prior to interaction or assessment by the nurse unless the client is unable to complete the questionnaire. The nurse may assist the client with the questionnaire if the client is visually impaired or requires assistance for any other reason.

After obtaining informed consent, clients attending the clinic were observed in their interaction with the nurse by the program evaluator. Clients were also asked by the evaluator to view their medical record, strictly for the purpose of extracting their scores on the MLHF. The MLHF (Appendix A) is a disease-specific 21-item measure of healthrelated quality of life (Rector \& Cohn, 1992). Patients with HF rate their perceptions about how much HF impacts their daily life, from 0 (not at all) to 5 (very much). Scores on the total instrument range from 0 to 105 , with higher scores reflective of worse perceived quality life. The MLHF has been shown to be highly reliable as demonstrated by the correlation ( $\mathrm{r}$ ) between repeated baseline assessments and measures of internal 
consistency such as Cronbach's alpha coefficient (a). In a study by Rector and Cohn (1992), construct validity was demonstrated by significant correlations of MLHF scores with NYHA functional classifications in 83 subjects with HF caused by left ventricular dysfunction $(r=0.80 ; \mathrm{p}=.01)$. Significant correlations were also noted between the MLHF and a single item measure that rated overall how much HF prevented them from living as they wanted in the past month $(r=0.80 ; \mathrm{p}=.01)$. Internal consistency reliability on the instrument was high, with Cronbach's alpha of 0.94 reported for the total scale. The Cronbach's alpha for the total scale in this study was 0.87 . Although there is some question that the questionnaire does not take into consideration the severity of the illness, overall it is proven to be an adequate assessment tool (Hak, Willems, Van der Wal, \& Visser, 2004).

When the client was called into the exam room, the nurse began her assessment, looking for signs of HF including shortness of breath with ambulation. Upon arrival to the exam room, the client was weighed and scores on the MLHF questionnaire were evaluated. Recommendations were made by the nurse based on the areas of the MLHF that the client scored higher on (which indicates lower quality). A physical assessment was obtained, including assessment of lung sounds, edema, and vital signs. If deemed necessary, a physician was available on site to provide expert consultation and medical triage. The clinic also provided intravenous diuretics as needed.

Newly diagnosed clients were provided with written instructions by the RN to help prevent HF and to assess themselves at home for signs of HF. The instructions included the following:

- Weigh yourself at the same time everyday and with the same scale 
- Take your medication exactly the way your doctor tells you to

- Do not add any salt to your foods

- $\quad$ Avoid eating prepared foods (for example, T.V. dinners, chips, canned soups)

1. Try to notice if you are getting more "winded" when you do activity that hasn't bothered you in the past

2. Has the amount of pillows that you sleep with changed?

3. Are your legs swelling?

4. Are your pants fitting tighter?

- If you should notice a weight gain of greater than 3 pounds over 1 to 2 days and/or you notice any of these items listed in 1-4, you should notify the clinic.

A one on one dietary consultation is also provided during a routine visit to the clinic. The nurse also provided the client with verbal and written information on sodium intake. The client was asked to discuss typical dietary intake during a week. One female client arrived at the clinic with journal entries including daily weights and daily intake. This had been suggested by the nurse on a previous consultation. Medications were also reviewed with clients to ensure appropriate dosing and allow for medication education. Any adjustments to medications were performed by the physician with nurse consultation.

Focusing on the evaluation and design. In order to focus on the program evaluation, a design plan was constructed and steps were developed to initiate the process. Prior to visiting the clinic, a series of questions were developed for the nurse interview. A one on 
one interview with the HF Clinic Coordinator/Patient Educator responsible for the clinic was conducted by the evaluator. An IRB approved consent form was reviewed with the $\mathrm{RN}$ prior to the interview. An in depth interview with the $\mathrm{RN}$ was conducted in order to better understand the role of the nurse, the components of the role, and what attributes contribute to success within the clinic. Specific information was addressed, such as the role of the nurse in the clinic, educational level, and years of experience. Data was recorded via hand written notes and reviewed by the interviewer. During the interview, discussion initiated with the nurse's title, educational background, and daily roles as previously discussed.

As the interview proceeded, a more in-depth interview focused on the nurse's philosophy of care as well as her insights as to factors that made the HF clinic successful. As the HF nurse reported, her philosophy of care was "to provide clients with the educational tools needed to make healthy decisions". "Each client is expected to take a role in their care; clients are responsible for refraining from sodium enriched foods, reporting symptoms of $\mathrm{HF}$ and monitoring daily weights". She described that "education is paramount" and that clients should be given the information needed in terms they understand and with that information the client can base decisions that will affect their health. "I can only provide the client with the information needed to help manage their HF symptoms; from there it is up to them".

When asked if she believed that nurse-led HF clinics are instrumental in achieving decreased re-hospitalization rates, the nurse replied:

"Absolutely! I know that we (as nurses) make a difference and our statistics prove that nurse-led clinics decrease re-hospitalization rates. Clients appreciate the extra time I, 
as a nurse, spend with them. During their clinic visits, education on diet, medication and the importance of early reporting of signs and symptoms of HF is reinforced. Just those extra few minutes that a physician may not have make a difference."

The nurse reported that the time spent with the clients made a difference and that it was advantageous to the program. She stated:

"Clients need to feel at ease and not rushed especially when I'm trying to teach about their health. I think that nurses have just a little more time to spend with the patients as compared to physicians. This gives the opportunity to develop a relationship with the patient. I also think that many patients are not as intimidated when speaking with a nurse versus speaking with a doctor."

The following questions related to what the nurse believed leads to a successful clinic and what personal characteristics are needed to accomplish this.

Question: What do you believe makes your clinic successful?

"The close monitoring and the aggressive outpatient treatment like when we monitor lab values, and when we administer intravenous diuretics. I also believe a rapport with the patients allows me a sense of when the patient 'really isn't feeling well.' For example, once you get to know a patient you can tell when they are not feeling well despite them saying 'I'm OK.' If you have built a relationship with the person you can then ask further questions to be able to determine whether or not they are OK or if they really are not feeling well. Some patients are very proud or may not want to be truthful for fear that they may end up in the hospital. Prior to coming to work at the clinic, I worked at the local hospital on a telemetry step down hospital unit. Many of 
the clients that I see at the HF clinic were admitted to my unit with HF. So when I came to work here, I already knew some of them pretty well. That helped too." Question: What are the main or most important personal characteristics for success in the field?

"Patience. Some of these patients require a lot of time. This could mean time spent with medication reconciliation, education, or time just to do 'hand holding'. Another important characteristic is empathy. If the nurse can build a rapport and trust with the patient then the patient will be more likely to be honest with how they are feeling. As I said previously, the nurse will also get to know the certain nuance of that particular patient and how they are truly feeling."

The nurse based the clinics' success on her relationship with the clients. This was evident during the observation of the nurse/client interaction. The clients seemed relaxed and compelled to tell the nurse about dietary mishaps. One client stated, "I know I shouldn't eat ham but yesterday I just had to have a ham sandwich". After providing the client with support and dietary substitutes, she explained to me that she can't expect every client to follow a strict diet but if she could explain to them why it is important to avoid sodium enriched foods than her job was completed.

Non-participatory observations were used to simply observe interactions between clients and nurses. A minimum of five client-nurse interactions were assessed. The focus of the observation was on the nature of the interaction and the how the client was assessed. Notes were recorded during the nurse-client clinic visits. Specific interests were placed on verbal communications and nonverbal behaviors. The data was then grouped and evaluated. 
During the nurse-client interactions, it was evident that the nurse was genuine and empathetic, demonstrating that she was able to understand the clients' feelings. She was very down to earth and appeared to have a close relationship with the clients. The nurse also allowed clients the opportunity to speak and played close attention to their concerns. Active listening was portrayed during all clinic visits. Any communication that occurred was provided in terms that the client was able to understand and it appeared that the use of medical terms was kept to a minimum. The nurse also verified that clients understood the information and education that was provided. Counseling was individualized to each client and based on their needs. During the clinic visit the nurse spent time evaluating the clients' dietary compliance, reiterating and discussing their daily weight monitoring and its significance to their treatment management. One role of the nurse is to involve the patient and their family members in their own plan of care therefore empowering the patient to assess his or her condition and make health promoting decisions. The nurse encouraged family to attend the clinic as the clients' support system and some clients attended the clinic visits with their caregivers or significant others. On one visit, a client stated that "I bring my wife because I can't remember everything you tell me".

When clients stated that they were unable to follow dietary restrictions, the nurse provided non-judgmental feedback. The nurse demonstrated understanding when dealing with the client's worries and concerns. She provided support and compassion. It was also apparent that she paid close attention to non-verbal cues as well as using the rapport she has with clients to investigate the clients health status. She would use techniques such as open ended questions, summarizing, and clarification to get the information needed to assess the client. Following the assessment of the client, clients were brought 
into her office and nurse was able to sit with the clients during the one on one interaction. This allowed for a more intimate interaction.

The interview and nurse-client interactions allowed for a greater understanding of the role and what key components are necessary for a successful HF clinic. Table 3 summarizes the HF clinic components and examples of the key functions of the nursing role as derived from the observation and interviews.

Gathering the credible evidence and justifying the conclusion. In order to ensure the program credibility, it was necessary to review the data provided by the clinic and compare to other data present in the literature. The HF clinic currently tracks clients' visits to the Emergency Room and admission/re-admission to the local hospital with a diagnosis of HF. Monthly, a report is run from within the clinic to determine how many of their patients were seen in the hospital with a diagnosis of HF. The outcome of HF readmission rates was provided by the clinic from their generated reports. This data was recorded by the site in the aggregate and represents de-identified aggregate data.

With permission from the program director, statistics related to re-hospitalization rates and ER visits were provided for the program evaluation. The data was compared to the national average of $24.5 \%$ as reported by Medicare figures for patients re-hospitalized with HF within a30 day period. Currently, the HF clinic reports a 30 day rehospitalization rate less than the national average, with a percentage of $18.6 \%$. This primary outcome variable was reported by the clinic as the six month average from June through December of 2009 month period. Although not encouraged by the evaluator, two of the five clients observed at the clinic stated they had not been hospitalized for over one year. 


\begin{tabular}{|c|c|}
\hline $\begin{array}{l}\text { Table } 3 . \\
\text { HF Clinic Components }\end{array}$ & Key Functions of Nursing Role \\
\hline Assessment & $\begin{array}{l}\text { - Nurse performs physical assessment at each clinic visit assessing } \\
\text { for signs/symptoms of HF and follows up with questioning client } \\
\text { Example. "You have gained two pounds since last week...do } \\
\text { you notice any differences in sleep patterns or daily } \\
\text { activities... do you find yourself more winded?...has the } \\
\text { amount of pillows that you sleep with changed?" } \\
\text { - Nurse uses the rapport she has with the patient to assess their } \\
\text { health status. } \\
\text { Example. "Once you get to know a patient you can tell when } \\
\text { they are not feeling well despite them saying 'I'm OK'." }\end{array}$ \\
\hline $\begin{array}{l}\text { Education for } \\
\text { patients/caregivers }\end{array}$ & $\begin{array}{l}\text { - Magnitude of Education provided to clients } \\
\text { Example. Written and verbal education provided and } \\
\text { reinforced at each clinic visit on their condition, dietary } \\
\text { restrictions, medications, signs and symptoms to report and } \\
\text { when to seek help } \\
\text { - Nurse provides education to clients while performing assessment } \\
\text { Example. "Just as we do here, it's important you weigh } \\
\text { yourself every day and call us if you have a weight gain of } \\
\text { three pounds in 1-2 days". } \\
\text { - Counseling of Sodium Restriction to client following a client's } \\
\text { statement "I know I shouldn't eat ham but yesterday I just had to } \\
\text { have a ham sandwich". } \\
\text { Example. Diet substitutions and how to assess sodiun on } \\
\text { Labels }\end{array}$ \\
\hline Quality of life & $\begin{array}{l}\text { - Recommendations provided to clients by nurse based on MLHF } \\
\text { scores as compared to previous clinic visits } \\
\text { Example. "I noticed that the side effects from your treatments } \\
\text { and your sleep pattern have caused you some } \\
\text { dissatisfaction...this is different from last time... Maybe you } \\
\text { can take your Lasix at } 4 \text { pm instead of } 6 \text { pm, it may help...not } \\
\text { causing you to wake up so many times in the night to use the } \\
\text { bathroom". }\end{array}$ \\
\hline Long term patient support & $\begin{array}{l}\text { - Medical, social and psychological support provided } \\
\text { - Family support encouraged by nurse } \\
\text { Example. Client reports "I get anxious thinking about how } \\
\text { much all my medications are going to cost and the office } \\
\text { visits...I don't make a lot of money" Nurse and clinic provide } \\
\text { clients with prescriptions with generic medications, resources } \\
\text { to prescription assistance programs and co-payment waived } \\
\text { for weekly appointments }\end{array}$ \\
\hline
\end{tabular}


With permission from the clients at the clinic, a chart review with specific interest on scores on the MLHF was conducted. Scores were assessed on five clients who had been attending the clinic at least six months. The clients who participated were of convenience in that they attended the clinic on the days that the evaluator conducted the observations. No other data was extracted from the clients' charts. This data was de-identified when it was recorded and was examined for trends within and between participants. The data was also compared to the norms of similar patients on the measure as described in the literature. The sample was not gender biased and consisted of both male and females attending the clinic. Of the five client scores evaluated at the clinic, each demonstrated improvement on questionnaire responses during the six month period. Overall, scores varied in improvement from 10-12 points at the six month mark $($ Table 4 ; lower score $=$ higher quality of life). Fluctuations in subcategories of the questionnaire did occur. One client's questionnaire demonstrated a higher score on the 'giving you side effects from treatments' question at six months than at the initial visit. As previously discussed, any concerns with the questionnaire subscales were discussed with the clients by the nurse at the clinic. The scores were compared to a randomized control study conducted by Kasper et al. (2002) that demonstrated an improvement of 12-13 points, on average, in the total MLHF scores. 


\section{Table 4.}

Patient scores on the MLHF questionnaire

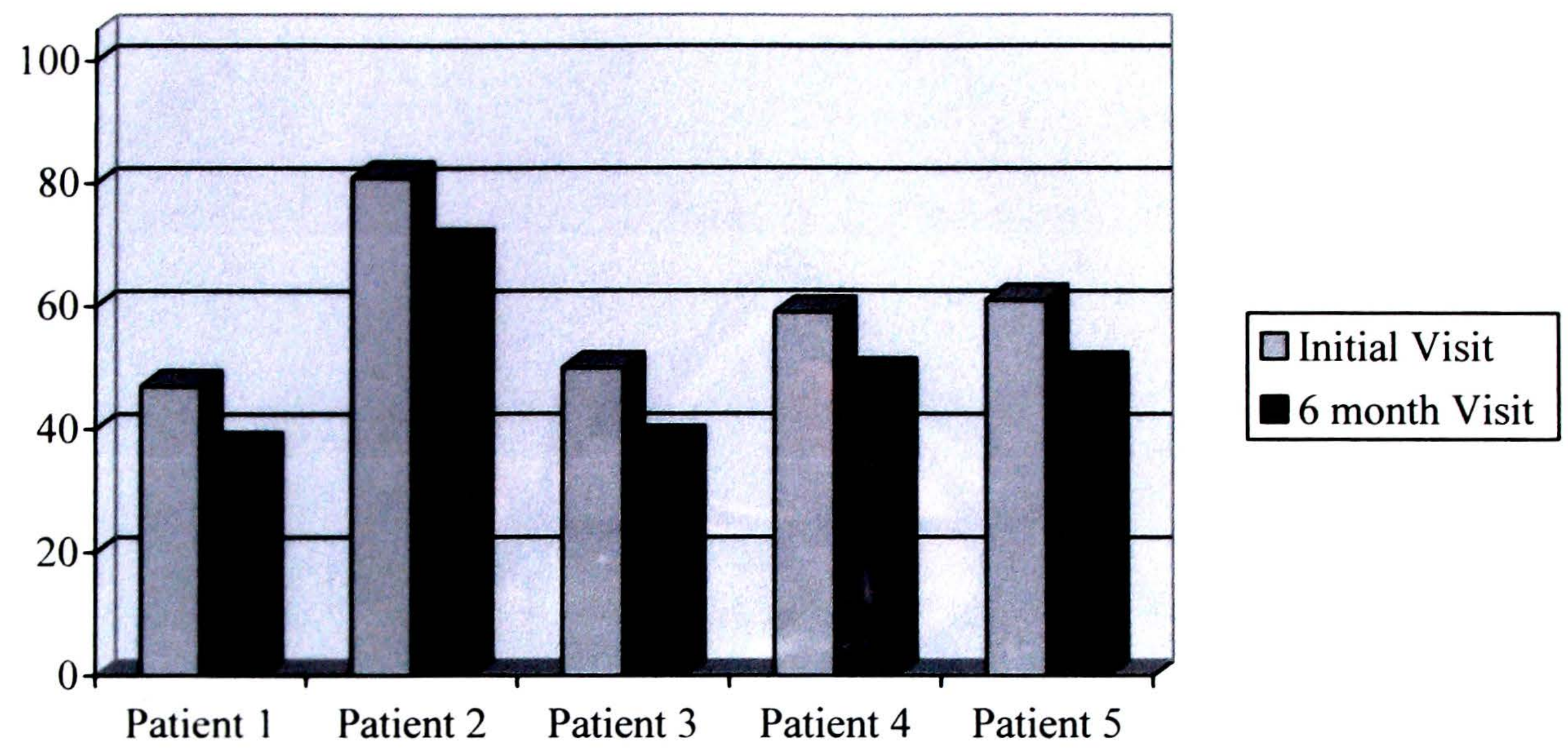

Ensure use of evaluation findings and share lessons learned. Ultimately, the

information gathered will be disseminated as the findings will be shared with the engaged stakeholders and presented to the nurse-led clinic providers. Since the target audience $f \circ r$ the study includes patients, physicians, nurses, and HF clinics, the best dissemination for the study will by journal publication. These findings will also be presented as part of graduate requirements to interested students and faculty. The main advantage of journal articles is the ease with which they can be accessed worldwide (Polit \& Beck, 2008). 
Summary and Conclusions

HF is a major and growing public health problem in the US (AHA, 2005). Clients with HF require extensive follow-up and education in order to prevent re-hospitalizations. Outpatient follow-up with nurse-led HF clinics appear to decrease readmission rates while increasing quality of life. The purpose of this project was to validate and possibly expand knowledge about specific aspects of the nursing role in particular that contribute to improved outcomes in nurse-led HF clinics. The HPM (Pender, 2006) was used to direct the project as it focuses on health promoting behaviors. Using the CDC framework (1999), a local HF clinic was evaluated. The program evaluation was guided by the six steps of the framework. The evaluators' observation of the interaction between the nurseclient and caregivers allowed for a better understanding of the nurses role. The nurse interview also assisted in this process by providing a view into her daily responsibilities and philosophy of care.

Along with the literature, nurse-led HF programs have been shown to improve patient outcomes. As previously mentioned, one of the primary goals of the clinic is to decrease the readmission rates and improve the quality of life for HF clients. This particular clinic demonstrated its success in significantly decreasing the number of unplanned rehospitalization rates and improving quality of life as measured by the MLHF questionnaire. Overall scores on the MLHF questionnaire improved over the six month period.

Education is essential in a successful HF program and is crucial in symptom management. The nurse-led HF clinic provides education and support to the clients at the clinic. The clinic reflects the qualities in the literature by providing clients with the 
education required to make health promoting decisions, long term patient support, and assessment of both physical and psychosocial aspects of the client. Ultimately, the HF program was a success due to the relationship amongst the nurse and the clients. This was evident as the clients were comfortable and able to express any concerns during clinic visits. The nurse was sincere and caring with her clients and demonstrated a true understanding of the clients' feelings. In conclusion, this program evaluation supported that nurse-led HF clinics are beneficial to improving quality of life and decreasing rehospitalization rates. 
Recommendations and Implications for Advanced Practice

Nurses have the ability to focus on the educational and clinical needs of the client as well as the supportive needs of the patient. They are the integral providers involved in educating, coaching, monitoring and supporting patients and their families during HF management. With this in mind, more attention needs to focus on the nurse's role and the specific components that contribute to a successful HF clinic. Besides focusing on the key components to the nurse's role, the nurse's educational background should also be assessed. Of the articles retrieved, there was minimal information in regards to the educational background of the nurses at the clinic. Because advanced practice nurses (APNs) are specially trained in evidence based practice and have a wealth of knowledge in disease management, they are the ideal professional to care for clients at a HF clinic. They also have the opportunity to help improve the quality of life for these patients and to decrease the economic burden on both the patients and the healthcare system. Since APNs are expert clinicians, HF clinics should require a minimum of Master's degree professionals to lead their clinics.

APNs can assess the signs and symptoms of cardiac destabilization, provide education, emotional support, counsel, assist in the development of health promoting behaviors, monitor therapy compliance and also act as the healthcare advocate for the patient and their caregivers. There is no question that APNs have the ability to care for HF clients on the outpatient setting. Further research needs to be conducted to determine if APN-led HF clinics are the most cost-effective and patient-focused method for HF management as compared to clinics led by other providers. 
HF clinics are specialized programs with multiple benefits for patients. With the evidence that demonstrates that $\mathrm{HF}$ clinics are advantageous to patients, it is important to refer these clients to HF clinics. Additional research should be performed to determine how many clients are referred to HF clinics prior to hospital discharge. The clinic must provide comprehensive education, and behavior modification strategies in order to improve HF management and improve patients' quality of life. In terms of policy, funding should be expanded for these clinics with reimbursement of certified APNs. With this in mind. clinic compensation will increase the likelihood that diverse enrollees are referred to the APN-led clinics while ensuring better access to their services. 


\section{References}

American Heart Association. (2005). Heart and Stroke Statistics: 2005 Update. Dallas, Texas: American Heart Association. Retrieved on October 11, 2007 from http://www.americanheart.org/statistics/coronary.html.

Becker M.H. (1974). The Health Belief Model and sick role behavior. In: Becker M.H., ed. The Health Belief Model: personal health behavior. Thorofare (NJ): Charles Slack; p. 82-93.

Centers for Disease Control \& Prevention (1999). Framework for program evaluation in public health. MMWR 48. 1-42. Retrieved on March 25, 2010 from http://www.cdc.gov/eval/framework.htm\#formats

Centers of Medicare \& Medicaid Services (2009). CMS Expands Information for Consumers about Outcomes of Care in America's Hospitals. Retrieved on July 31, 2009 from http://www.cms.gov/apps/media/press/factsheet.asp

Cline C.M., Broms K., \& Willenheimer R.B. (1996). Hospitalization and health care costs due to congestive heart failure in the elderly. American Journal of Geriatric Cardiology, 5, 10-23.

Cline C.M., Israelsson B.Y., Willenheimer R.B., Broms K., \& Erhardt L.R. (1998). Cost effective management programme for heart failure reduces hospitalization. Heart, $80,442-446$.

Copstead, L. C., \& Banasik, J. L. (2000). Pathophysiology: Biological and Behavioral Perspectives ( $2^{\text {nd }}$ ed.). Philadelphia, PA: W.B. Saunders Company. 
Ducharme A., Deyon O., White M., Rouleau J.L., \& Brophy J.M. (2005). Impact of a multidisciplinary CHF clinic: a randomized trial. CMA, 173 (1), 40-45 Retrieved on October 11, 2007 from www.pubmedcentral.nih.gov/articlerender.fcgi?artid= 1167811.

Hak, T., Willems, D., Van der Wal, G., \& Visser F. (2004). A qualitative validation of the Minnesota Living with Heart Failure Questionnaire. Quality of Life Research, 13: $417-426$

Kasper E. K., Gerstenblith G., Hefter G., Van Anden E., Brinker J. A., Thiemann D. R., Terrin M., Forman S., \& Gottlieb S. H. (2002). A randomized trial of the efficacy of multidisciplinary care in heart failure outpatients at high risk of hospital readmission. Journal of American College of Cardiology, 39, 471-480

McDonald K., Ledwidge M., Cahill J., Quigley, P., Maurer, B., Travers, B., Ryder, M., Kieran, E., Timmons, L., \& Ryan, E. (2002). Heart failure management: multidisciplinary care has intrinsic benefit above the optimizations of optimization of medical care. Journal Card Fail., 8,142-148.

Pender, N.J. (1974). Patient identification of health information received during hospitalization. Nursing Research, 23, 262-267.

Pender, N.J., Murdaugh, C. L., \& Parsons, M.A. (2002). Health Promotion in Nursing Practice (4 ${ }^{\text {th }}$ Edition). Upper Saddle River, NJ: Prentice Hall.

Pender, N. J., Murdaugh, C., \& Parsons, M.A. (2006). Health Promotion in Nursing Practice, (5th edition). Upper Saddle River, NJ: Prentice-Hall

Polit D., \& Beck C. (2008). Nursing Research: Generating and Assessing Evidence for Nursing Practice $\left(8^{\text {th }}\right.$ ed.). Philadelphia, PA: Lippincott Williams and Wilkins. 
Rector T.S., \& Cohn J.N. (1992). Assessment of patient outcome with the Minnesota Living with Heart Failure Questionnaire: reliability and validity during a randomized, double-blind, placebo controlled trial of pimobendan. American Heart Journal, 124, 1017-1025.

Rich M.W., Beckham V., Wittenberg C., Leen C., Freedland K.E., \& Carney R.M. (1995). A multidisciplinary intervention to prevent readmission of elderly patients with heart failure. New England Journal of Medicine, 333, 1190-1195.

Stromberg A., Martensson J., Frindlund B., Levin L. A., Karlsson J. E., \& Dahlstrom U. (2003). Nurse-led heart failure clinics improve survival and self-care behavior in patients with heart failure. European Heart Journal, 24, 1014-1023. Retrieved on October 11, 2007 from http://eurheart.oxfordjournals.org/cgi/reprint/24/11/1014 
Appendix A

Minnesota Living with Heart Failure Questionnaire 


\section{MINNESOTA LIVING WITH HEART FAILURE ${ }^{\circledR}$ QUESTIONNAIRE}

These questions concern how your heart failure (heart condition) has prevented you from living as you wanted during the last month. The items listed below describe different ways some people are affected. If you are sure an item does not apply to you or is not related to your heart failure then circle 0 (No) and go on to the next item. If an item does apply to you, then circle the number rating how much it prevented you from living as you wanted. Remember to think about ONLY THE LAST MONTH.

Did your heart failure prevent you

from living as you wanted during

the past month ( 4 weeks) by -

\begin{tabular}{lll} 
& Very & Very \\
No & Little & Much \\
\hline
\end{tabular}

\begin{tabular}{|c|c|c|c|c|c|c|}
\hline 1 Causing swelling in your ankles or legs? & 0 & 1 & 2 & 3 & 4 & 5 \\
\hline 2 Making you sit or lie down to rest during the day? & 0 & 1 & 2 & 3 & 4 & 5 \\
\hline 3 Making your walking about or climbing stairs difficult? & 0 & 1 & 2 & 3 & 4 & 5 \\
\hline 4 Making your working around the house or yard difficult? & 0 & 1 & 2 & 3 & 4 & 5 \\
\hline 5 Making your going places away from home difficult? & 0 & 1 & 2 & 3 & 4 & 5 \\
\hline 6 Making your sleeping well at night difficult? & 0 & 1 & 2 & 3 & 4 & 5 \\
\hline $\begin{array}{l}7 \text { Making your relating to or doing things with your friends or } \\
\text { family difficult? }\end{array}$ & 0 & 1 & 2 & 3 & 4 & 5 \\
\hline 8 Making your working to earn a living difficult? & 0 & 1 & 2 & 3 & 4 & 5 \\
\hline \begin{tabular}{l|l}
9 & Making your recreational pastimes, sports or hobbies \\
difficult?
\end{tabular} & 0 & 1 & 2 & 3 & 4 & 5 \\
\hline 10 Making your sexual activities difficult? & 0 & 1 & 2 & 3 & 4 & 5 \\
\hline 11 Making you eat less of the foods you like? & 0 & 1 & 2 & 3 & 4 & 5 \\
\hline 12 Making you short of breath? & 0 & 1 & 2 & 3 & 4 & 5 \\
\hline 13 Making you tired, fatigued, or low on energy? & 0 & 1 & 2 & 3 & 4 & 5 \\
\hline 14 Making you stay in a hospital? & 0 & 1 & 2 & 3 & 4 & 5 \\
\hline 15 Costing you money for medical care? & 0 & 1 & 2 & 3 & 4 & 5 \\
\hline 16 Giving you side effects from treatments? & 0 & 1 & 2 & 3 & 4 & 5 \\
\hline 17 Making you feel you are a burden to your family or friends? & 0 & 1 & 2 & 3 & 4 & 5 \\
\hline 18 $\quad$ Making you feel a loss of self-control in your life? & 0 & 1 & 2 & 3 & 4 & 5 \\
\hline 19 Making you worry? & 0 & 1 & 2 & 3 & 4 & 5 \\
\hline $\begin{array}{l}20 \text { Making it difficult for you to concentrate or remember } \\
\text { things? }\end{array}$ & 0 & 1 & 2 & 3 & 4 & 5 \\
\hline $21 \mid$ Making you feel depressed? & 0 & 1 & 2 & 3 & 4 & 5 \\
\hline
\end{tabular}




\section{Michael Savard}

From: $\quad$ "George Gingell" <George.Gingell@HarveyBP.com>

To: <trimman78@comcast.net>

Sent: Monday, May 03, 2010 11:31 AM

Subject: susan walker

\section{QUOTE ONLY}

White garden window standard buck size $40^{\prime \prime} \times 40^{\prime \prime}$

White frame $49 / 16$ jamb

1 "low e argon gas

Tempered top glass

Insulated seatboard

- Nail fin and flashing included

Thanks george

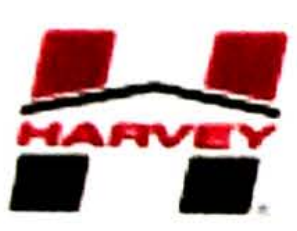

HARVEY 